\title{
Pupil cycle time: a simple way of measuring an autonomic reflex
}

\author{
CN MARTYN, DJ EWING \\ From the University Department of Medicine, Royal Infirmary, Edinburgh, UK
}

SUMmary Persistent regular oscillations of the pupil of the eye can be induced using a slit-lamp. The period of these oscillations, pupil cycle time (PCT), is simple to measure with a hand-held stopwatch. Evidence from pharmacological testing suggests that PCT is a sensitive measure of dysfunction of the parasympathetic efferent limb of the pupillary light reflex arc. This is confirmed by the finding that PCT is prolonged in a high proportion of patients with evidence of autonomic neuropathy judged by abnormalities of their cardiovascular reflexes. The method provides a simple, non-invasive way of quantifying an autonomic reflex and it may be of value in investigating patients with diseases affecting the autonomic nervous system.

Almost every organ system of the body possesses an autonomic innervation and the contribution of autonomic dysfunction to the symptomatology of many diseases is being increasingly recognised. However, the only simple tests of autonomic function available to the practising clinician depend on measurements of cardiovascular reflexes ${ }^{12}$ as investigation of other systems requires elaborate apparatus and is often unpleasant for the patient. In this paper we describe a simple method of quantifying the pupillary light reflex and its application to the diagnosis of autonomic neuropathy.

In 1944 Stern described how persistent oscillations of the pupil could be induced using a slit-lamp. ${ }^{3}$ A fine pin-point of light was projected through the pupil near the margin of the iris thus stimulating the pupillary light reflex. As the pupil constricted, the iris margin moved towards the centre of the pupil and occluded the beam of light. Because no light now reached the retina, the stimulus for pupillary constriction was removed. The pupil therefore dilated, which re-exposed the retina to the beam of light and re-started the cycle of constriction and dilatation. Miller and Thomson ${ }^{4}$ improved the technique and demonstrated the stability and repeatability of measurements of the period of the pupillary oscillations, which they dubbed pupil cycle time (PCT). They applied the method, with limited success, to the detection of optic nerve pathology. ${ }^{5}$

Our study concerns the applications of mea-

Address for reprint requests: Dr CN Martyn, MRC Environmental Epidemiology Unit, Southampton General Hospital, Southampton, SO9 4XY, UK.

Received 24 September 1985. Accepted 2 November 1985 surement of PCT to the detection of dysfunction in the efferent autonomic limb of the reflex pathway. We have measured PCT in normal subjects, diabetics and patients with progressive autonomic failure and compared the results with those obtained by testing of cardiovascular autonomic reflexes.

\section{Patients and methods}

\section{Patients}

Fifty normal subjects (mean age 34 years, range 19-65) drawn from laboratory staff and hospital out-patients free from both ocular disease and disorders known to affect autonomic function had PCT measured in both eyes. No subject was taking drugs which could have affected autonomic function. In 135 diabetic patients (mean age $\mathbf{4 0}$ years, range 18-64) measurements of PCT and five cardiovascular autonomic reflexes were carried out. PCT was measured by an observer who was unaware of the results of cardiovascular reflex testing. Patients who had required treatment for retinopathy within the previous 6 months were excluded. Six patients with progressive autonomic failure were also studied; details of this group are included in table 1.

\section{Pupil cycle time}

The method is derived from that of Miller and Thomson ${ }^{4}$ and differs only in minor detail. The subject sits at the slitlamp in a dimly lit room and is instructed to look towards the light source of the slit-lamp. A narrow $(0.5 \mathrm{~mm})$ horizontally-orientated slit of light is focussed accurately on the lower part of the iris in a plane perpendicular to it. The beam of light is slowly elevated until it just impinges on the pupil (fig 1). In normal subjects this invariably initiates the cycle of constriction and dilatation described in the introduction. The pupillary oscillations are easily observed through the low-power binocular microscope of the slitlamp and the time for 100 cycles of dilatation and constriction can be measured with a hand-held stop-watch. Two 
Table 1 Pupil cycle time ( $\mathrm{ms}$ ) in patients with progressive autonomic failure

\begin{tabular}{|c|c|c|c|c|}
\hline Patient & Age $(y r)$ & Sex & $P C T($ left $)$ & $P C T$ (right) \\
\hline $\begin{array}{l}1 \\
2 \\
3 \\
4 \\
5 \\
6\end{array}$ & $\begin{array}{l}64 \\
55 \\
46 \\
66 \\
62 \\
60\end{array}$ & $\begin{array}{l}M \\
M \\
F \\
F \\
M \\
M\end{array}$ & $\begin{array}{l}1327 \\
2377 \\
1751 \\
1750 \\
\text { Corneal opacity } \\
2374\end{array}$ & $\begin{array}{l}1219 \\
\text { No cycling } \\
1311 \\
\text { No cycling } \\
1287 \\
2200\end{array}$ \\
\hline
\end{tabular}

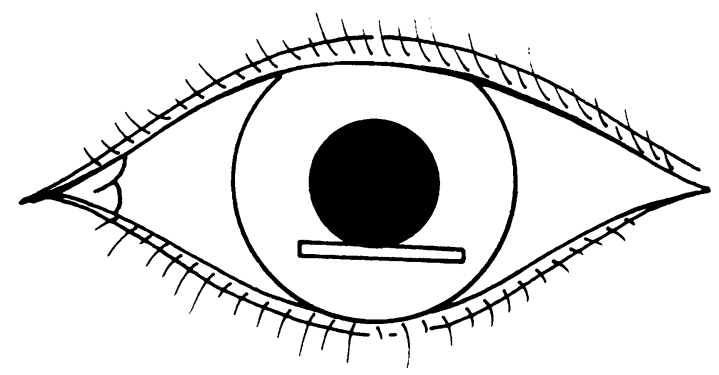

Fig 1 Diagram showing position of the stimulating slit of light in relation to the pupil.

runs of 30 and one of 40 cycles are timed. The mean PCT is then calculated by simple division. PCT was never identical in the two eyes of a single subject and for the purposes of analysis we have considered only the longer of the two PCT estimates from each subject.

Cardiovascular autonomic tests

Five tests of autonomic cardiovascular reflexes were used. These tests: heart rate changes after a Valsalva manoeuvre, with deep breathing, and when moving from a lying to a standing position and blood pressure changes in response to standing and sustained handgrip are described in detail elsewhere. $^{1}$

\section{Results}

Mean $( \pm S D)$ PCT in this group of 50 normal subjects was $946 \pm 120 \mathrm{~ms}$. We derived a value of $1150 \mathrm{~ms}$ as the upper limit of normal by estimating the 95th centile using Student's $t$ distribution and a log transformation of the data. There was no significant correlation with age in normal subjects $(r=0 \cdot 18)$. The width of the illuminating slit, time of day that the measurement was performed and the duration of dark adaption before measurements were made had no effect on PCT. There was no consistent difference between left eye and right eye and no evidence that PCT became prolonged with repeated measurements.

Repeatability of the test was established by measuring 20 subjects twice at an interval of between one week and one month. The coefficient of variation of the method was $4 \cdot 2 \%$.

Table 2 and fig 2 show the effects of two doses $(0 \cdot 1$ and $0.3 \mathrm{mg}$ ) of intravenous atropine on PCT in a normal subject. The doses were too small to produce a rise in heart rate but an immediate lengthening of PCT was apparent. This indicates that the measurement of PCT is very sensitive to minor degrees of parasympathetic blockade. Intraocular homatropine $(0.4 \%)$ or tropicamide $(1 \%)$ produced a lengthening of pupil cycle time within the first few minutes. After 10-15 minutes oscillations of the pupil were abolished entirely. Sympathetic blockade by intraocular instillation of guanethidine $(4 \%)$ had no significant effect on PCT despite a marked degree of miosis. The treatment was continued twice daily for 3 days in one subject but no effect on PCT was observed even at the end of this period.

Sympathetic stimulation by the instillation of phenylephrine $(10 \%)$ eye drops was without effect on PCT although the amplitude of the pupillary excursions was reduced. The results of pharmacological testing are summarised in table 2.

The diabetics were classified into three groups according to the results of the cardiovascular reflex tests: (a) patients in whom all five tests were normal, (b) patients in whom one or two tests were abnormal and (c) patients in whom three or more tests were abnormal. Mean PCT for these groups became

Table 2 Effect of drugs on pupil cycle time

\begin{tabular}{|c|c|c|c|}
\hline Drug & Route & Number of subjects & Effect on PCT \\
\hline $\begin{array}{l}\text { Atropine } 0.1 \mathrm{mg} \\
\text { Atropine } 0.3 \mathrm{mg} \\
\text { Homatropine }(1 \%) \\
\text { Tropicamide }(0.5 \%) \\
\text { Phenylephrine }(10 \%) \\
\text { Guanethidine }(5 \%)\end{array}$ & $\begin{array}{l}\text { iv } \\
\text { iv } \\
\text { Intra-ocular } \\
, ", ", \\
", \quad,\end{array}$ & $\begin{array}{l}1 \\
1 \\
2 \\
3 \\
3 \\
2\end{array}$ & 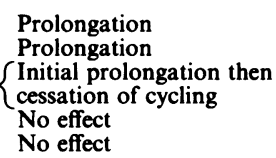 \\
\hline
\end{tabular}



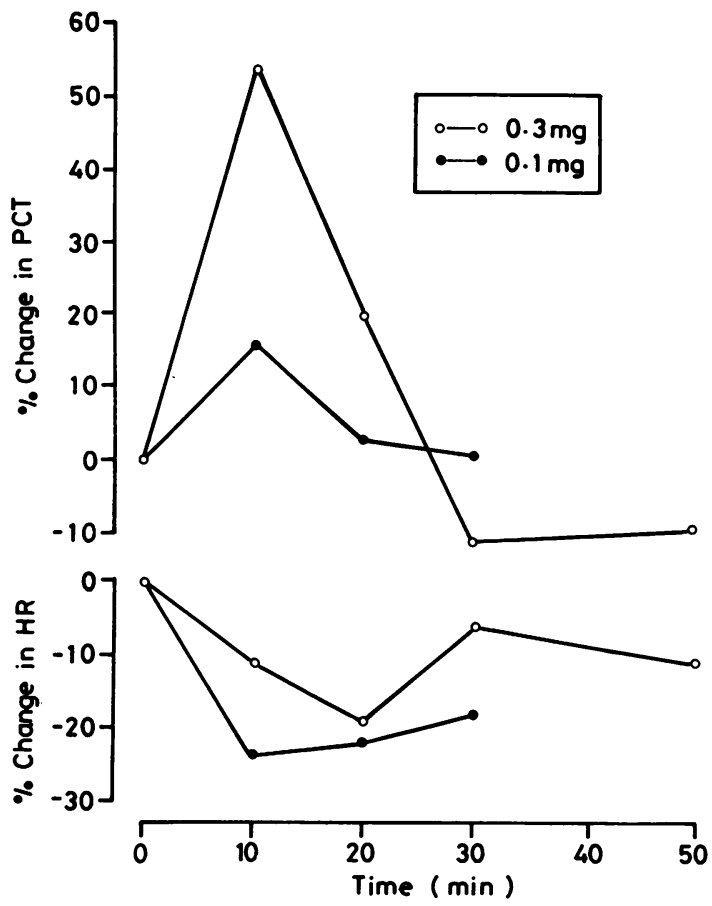

Fig 2 The effect of two doses of intravenous atropine on pupil cycle time and heart rate in a normal subject.

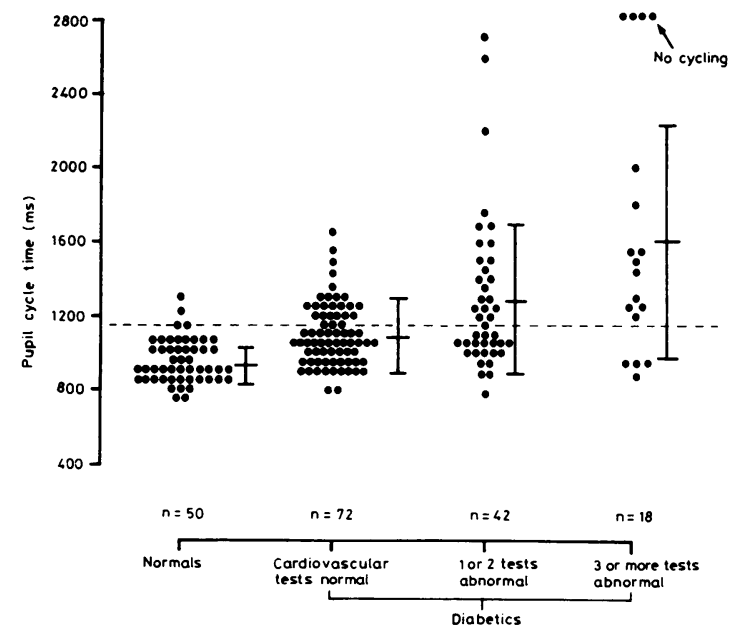

Fig 3 Pupil cycle times in normal subjects and diabetics. Diabetics are grouped according to the number of abnormal results found on cardiovascular reflex testing. Means $\pm S D$ are indicated. The upper limit of normal is shown by the dotted line. progressively longer as the number of cardiovascular reflex abnormalities increased (see fig 3). Analysis of variance demonstrates significant differences between groups $(\mathrm{p}<0.001)$.

The results of measurement of PCT in patients with progressive autonomic failure are summarised in table 1. Each patient had abnormalities of all five cardiovascular reflex tests. None had abnormal pupil light reflexes as determined by the normal technique of clinical examination but PCT was prolonged in all patients. In some it proved impossible to induce persistent pupillary oscillation at all.

\section{Discussion}

The period of pupillary oscillations induced by this method depends on the structural and functional integrity of all components of the pupillary light reflex arc. Theoretically, at least, retinal function, speed and pattern of impulses reaching the mid-brain from the optic nerve, synaptic transmission in the mid brain, efferent nerve activity and function of the smooth muscle of the iris are all potentially important factors determining PCT. Nonetheless, it seems that lengthening of PCT is much more likely to occur when defects are present in the efferent limb of the reflex arc than when they are present in the afferent limb. The evidence for this is partly pharmacological in that very small doses of atropine given intravenously or homatropine or tropicamide instilled intra-ocularly result in an immediate and dramatic lengthening of PCT. Other evidence comes from the experience of Cox et al who used a nearly identical method to investigate patients with a history of optic neuritis. ${ }^{5}$ They found the method to be far less sensitive in detecting optic nerve pathology than measurement of visually evoked responses or the quantitative detection of a relative afferent pupillary defect. We have found, as did Miller and Thomson, ${ }^{4}$ that PCT is independent of the brightness of the stimulating light source over a wide range of intensity. This also makes it unlikely that retinal and optic nerve function, unless profoundly impaired, contribute much to lengthening of PCT.

Defects of contraction of the iris smooth muscle itself might cause PCT to be prolonged. Ultrastructural studies have demonstrated abnormalities in the muscle of the diabetic iris though these may be consequent on damage to the innervating nerve fibres. Degenerating autonomic nerve endings in the iris have been observed. ${ }^{6}$ The diabetic iris actually shows an increased response to both cholinomimetic and sympathomimetic drugs $^{7-9}$ which indicates denervation hypersensitivity. It is more likely that prolongation of PCT is caused by abnormality in the 
innervation of the iris than abnormality in the smooth muscle itself.

We have been able to show that prolongation of pupil cycle time in diabetics correlates well with evidence of autonomic neuropathy obtained from testing of cardiovascular reflexes. Patients whose cardiovascular reflexes are very abnormal tend also to have pupils which cycle slowly. The association is not perfect and we have discovered patients with normal cardiovascular reflexes and long PCT and vice versa. If the results of cardiovascular reflex testing are taken as an indication of whether autonomic dysfunction is present or not, the sensitivity of PCT as a diagnostic test is $59 \%$ and the specificity $70 \%$. However, abnormalities in cardiovascular reflex tests do not necessarily reflect autonomic dysfunction in other systems and tests based on these reflexes cannot be considered as the gold standard against which to judge other measures of autonomic function. The imperfect correlation of these tests with measurements of PCT can also be interpreted as evidence that autonomic dysfunction in different organ systems does not occur simultaneously.

The association of pupillary abnormalities with autonomic and peripheral nerve dysfunction in diabetics has been demonstrated previously using pupillographic techniques. ${ }^{1011}$ Our intention in this paper is not to reiterate this observation but to draw attention to the iris as an easily observed structure innervated exclusively by the autonomic nervous system and to describe how measurement of PCT provides a simple way of quantifying one aspect of its function. Our investigations suggest that measurement of PCT is particularly sensitive to dysfunction in the parasympathetic efferent limb of the pupillary light reflex arc. We believe that measurement of PCT will provide a useful addition to existing tests of autonomic function, which at present are almost entirely directed towards the measurement of cardiovascular reflexes, in the diagnosis and investigation of patients with diseases affecting the autonomic nervous system.

We are grateful to Carol O'Neill and Kate Henderson for expert technical help. Dr DJ Ewing is a Wellcome Trust Senior Lecturer.

\section{References}

${ }^{1}$ Ewing DJ, Clarke BF. Diagnosis and management of diabetic autonomic neuropathy. $\mathrm{Br}$ Med J 1982;285: 916-8.

${ }^{2}$ Bennett T. Physiological investigation of diabetic autonomic failure. In: Bannister R, ed. Autonomic Failure. Oxford University Press, 1983:406-36.

${ }^{3}$ Stern HJ. A simple method for the early diagnosis of abnormality of the pupillary reaction. $\mathrm{Br} J$ Ophthalmol 1944;28:275-6.

${ }^{4}$ Miller SD, Thomson HS. Edge-light pupil cycle time. $\mathrm{Br} J$ Ophthalmol 1978;62:495-500.

${ }^{5}$ Cox TA, Thomson HS, Hayreh SS, Snyder JE. Visual evoked potential and pupillary signs. Arch Ophthalmol 1982;100:1603-7.

${ }^{6}$ Fujii T, Ishikawa $S$, Uga S. Ultrastructure of iris muscles in diabetes mellitus. Ophthalmologica 1977;174:228-39.

${ }^{7}$ Sigsbee B, Torkelson R, Kadis G, Wright JW, Reeves AG. Parasympathetic denervation of the iris in diabetes mellitus. J Neurol Neurosurg Psychiatry 1974;37:1031-5.

${ }^{8}$ Smith SA, Smith SE. Evidence for a neuropathic aetiology in the small pupils of diabetes mellitus. Br J Ophthalmol 1983;67:89-93.

${ }^{9}$ Hayashi M, Ishikawa S. Pharmacology of pupillary responses in diabetics. Jpn J Ophthalmol 1979;23:65-72.

${ }^{10}$ Friedman SA, Feinberg R, Podolak E, Bedell RHS. Pupillary abnormalities in diabetic neuropathy: a preliminary study. Ann Intern Med 1967;67:977-83.

${ }^{11}$ Smith SE, Smith SA, Brown PM, Fox C, Sonksen PH. Pupillary signs in diabetic autonomic neuropathy. $\mathrm{Br}$ Med J 1978;2:924-7. 